\title{
The comparison of two different intraarticular injections using a sonographic anterolateral approach in patients with osteoarthritic knee
}

\author{
${ }^{1}$ Department of Anesthesiology and Pain Medicine, Chonbuk National University Medical School and Hospital, \\ ${ }^{2}$ Department of Preventive Medicine, Chonbuk National University Medical School, \\ ${ }^{3}$ Department of Oral and Maxillofacial Surgery, Chonbuk National University School of Dentistry, Jeonju, Korea
}

Jin Wook Choi ${ }^{1}$, Jun Ho Lee', Minjong $\mathrm{Ki}^{1}$, Myung Jong Kim ${ }^{1}$, Sehrin Kang ${ }^{1}$, Juhyung Lee ${ }^{2}$, Jun-Rae Lee, ${ }^{3}$, Young-Jin Han', and Ji-Seon Son ${ }^{1}$

\begin{abstract}
Background: The intraarticular (IA) injection has become popular for the management of the osteoarthritic knee without an effusion. The success rate of IA injection would be better if it was able to be visually confirmed. We hypothesized that an anterolateral approach, which targets the synovial membrane of the lateral condyle using ultrasound, would provide an equivalent alternative to the anterolateral approach, targeting the synovial membrane of the medial condyle for IA injection of the knee.
\end{abstract}

Methods: A total of 96 knees with osteoarthritis were randomized placed into the two groups, which were group I (anterolateral approach to the medial condyle) and group II (anterolateral approach to the lateral condyle). The primary outcome was to compare the success rate of the two methods of IA injection. The required length of the needle for injection was also measured and compared. Pain intensity was assessed using the Numeric Rating Scale in order to evaluate the success of injection.

Results: There were no significant differences in the success rate between both groups. The success rate of group I and group II were $87.8 \%$ (95\%, CI 78.7-97.0) and 91.5\% (95\%, CI 83.6-99.5), respectively $(P=0.549)$. The needle depth was $5.0 \pm 0.8(3.0$ to $6.1 \mathrm{~cm})$ in group I, and $3.0 \pm 0.8(1.5$ to $5 \mathrm{~cm})$ in group II $(P<$ $0.001)$.

Conclusions: The anterolateral approach to the lateral femoral condyle, using ultrasound, is an alternative method to the approach targeting the medial femoral condyle, using shorter needle. (Korean J Pain 2018; 31: 289-95)

Key Words: Epiphyses; Femoral condyle; Intraarticular injections; Knee joint; Needle; Osteoarthritis; Synovial membrane; Ultrasound.

Received August 6, 2018. Revised September 20, 2018. Accepted September 25, 2018.

Correspondence to: Ji-Seon Son

Department of Anesthesiology and Pain Medicine, Chonbuk National University Hospital, 20 Geonji-ro, Deokjin-gu, Jeonju 54907, Korea Tel: +82-63-250-1246, Fax: +82-63-250-1241, E-mail: sjs6803@chonbuk.ac.kr

() This is an open-access article distributed under the terms of the Creative Commons Attribution Non-Commercial License (http:// creativecommons.org/licenses/by-nc/4.0/), which permits unrestricted non-commercial use, distribution, and reproduction in any medium, provided the original work is properly cited.

Copyright (C) The Korean Pain Society, 2018 


\section{INTRODUCTION}

Intraarticular (IA) injection with hyaluronic acid or a steroid has become popular for the management of the osteoarthritic knee [1-3]. A number of anatomic landmarkguided IA injections of the knee have been used, including the lateral or medial midpatellar approaches, and the lateral or medial anterior approaches [4,5]. The target of the IA injection using an anterolateral and anteromedial approaches, with the patient in the sitting or supine position with the knee bent, has been traditionally the synovial membrane reflections in the intercondylar notch, but the success rate is only 71 and $75 \%$, respectively [5]. Although an IA injection of the knee is not a complicated procedure, it could be difficult to succeed blindly. In a "dry" joint without an effusion, IA placement of the needle using an anterolateral or anteromedial approaches may be difficult blindly.

The success rate of IA injection would be better if it is able to be visually confirmed. Ultrasound methods have been attempted for accurate intraarticular injection of the knee [6-8]. In a few studies, the needle was inserted at the synovial membrane of the medial femoral condyle using an ultrasound for IA injection [6,7]. The study showed that ultrasound guided IA injection with anterolateral portals was $97 \%$ accurate, and an equivalent alternative to the standard lateral midpatellar portal [6]. However, this method was clinically uncomfortable, because of the following reasons. First, it requires a needle longer than 5 $\mathrm{cm}$ long, which has to be manufactured by the order. Second, it is difficult to perform the in-plane technique easily because of the long distance between the probe and the site of needle insertion. Repositioning of the probe for a better sonographic image results in missing the shade of the femoral condyle, so the needle has to be repositioned in many cases.

We hypothesized that an anterolateral approach targeting the lateral femoral condyle would provide an equivalent success rate to the anterolateral approach to the medial femoral condyle, using a shorter needle when performing the injection with in-plane technique.

\section{MATERIALS AND METHODS}

The protocol was approved by the Institutional Review Board of Chonbuk National University Hospital. Additional- ly, written informed consent was obtained from all patients before the beginning of the study. The criteria for the study included patients who had been presented and diagnosed with osteoarthritis of the knee, with persistent and significant pain in the involved joint with a Numeric Rating Scale (NRS) score $\geq 4$ out of 10 , and the recommendation from the physician for an IA injection. Exclusion criteria were KL grade 4 osteoarthritis, the presence of infection, the use of warfarin therapy, allergy to the local anesthetics, or a body mass index (BMI) of more than 40 $\mathrm{kg} / \mathrm{m}^{2}$. In this case, the referred patients underwent a preoperative ultrasound examination to identify "dry" knee without a clinically detectable effusion.

The sample size calculations were performed using the PASS (PASS 14 Power Analysis and Sample Size Software (2015). NCSS, LLC. Kaysville, UT) based on the reference value (0.97), non-inferiority margin value (0.85), beta (0.2), and alpha value (0.05) found in the pilot study. A total sample size was calculated to be 96 participants.

In the study, 96 knees were enrolled. The participants were randomly assigned to one of two groups which were group I (anterolateral approach to the medial condyle, 49 knees) and group II (anterolateral approach to the lateral condyle, 47 knees). Although the two groups were randomly assigned to in equal numbers, a different approach was applied for one participant unintentionally.

IA injection in this study was basically performed under ultrasound guidance. The position of the patient was supine, with a 90 degree flexion of the knee. The anterolateral approach is a method of injection on the anterolateral portal. The anterolateral portal was defined by the adjoining structures of the inferolateral border of the patellar, the lateral tibial plateau, and the infrapatellar tendon (Fig. 1).

The entry point of the needle was about $1.5 \mathrm{~cm}$ from the lateral margin of the infrapatellar tendon. The intended needle target points were palpated and marked after getting clear femoral condylar imaging (Fig 2A and B). Once the needle was visualized, it was advanced toward the central point of the image of the femoral condyle, which was made visible by adjusting the tilt of the probe. A $7 \mathrm{~cm} 25$ gauge (G) needle with a $30 \mathrm{~cm}$ injecting line was used for this procedure. In group I, the target of the needle was the synovial membrane covering the medial femoral condyle $[6,7]$. In group II, the target of the needle was the synovial membrane covering the lateral femoral condyle. 


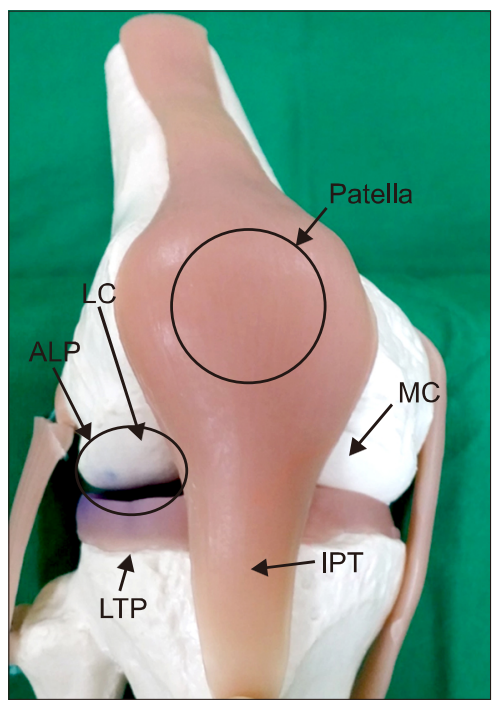

Fig. 1. Anatomical representation using a plastic model. This is a reproduced knee with 90-degree flexion in the supine position. Anatomic landmarks are marked on the photograph. Anterolateral portal (ALP) is presented in a small circle. LC: lateral femoral condyle, MC: medial femoral condyle, IPT: infrapatellar tendon, LTP: lateral tibial plateau.

An ultrasound unit with a 13-6 MHz linear array transducer (Edge ${ }^{\circledR}$, Sonosite Inc., Bothell, WA) was used to determine the location of the femoral condyle, the position of the needle tip, and the intraarticular and the extraarticular fluid flow.

True IA injection was determined by observing 1) the demonstration of the free flow of fluid from the needle tip into the intraarticular space, and 2) the demonstration of dilation of the intraarticular space with the injected fluid $[7,8]$. Extraarticular injection was determined by observing 1) fluid movement into the anterior fat pad or the backflow of fluid along the needle shaft, 2) increased echogenicity in the septa of the anterior fat pad, and 3) the lack of fluid movement into and dilation of the IA space [7].

In group I (the anterolateral approach to the medial femoral condyle), the needle passed through the anterior fat pad under the infrapatellar tendon from the anterolateral portal, until the tip of the needle penetrated the synovial membrane covering the medial condyle (Fig. 2A). The pain physician placed the ultrasound probe over the anteromedial portion of the bent knee, and thus approximated 90 degrees to the long axis of the needle. The needle was advanced to the central point of targeted medial femoral condyle, which was visualized by adjusting the tilt of the probe. In group II (the anterolateral approach to the lateral femoral condyle), the needle passed through the anterior fat pad from the anterolateral portal, until the tip of the needle penetrated the synovial membrane covering the lateral condyle (Fig. 2B). The pain physician carefully placed the ultrasound probe over the central portion of the tibial plateau, and then caudally tilted it until a clear image of the lateral condyle was seen. The needle was advanced to the central point of the targeted lateral femoral condyle, which was clearly visualized by adjusting the tilt of the probe. The entry point of needle was about $1.5 \mathrm{~cm}$ from the lateral margin of the infrapatellar tendon, similar to group I.

The skin around the injection site was prepared with Betadine (povidone-iodine), and surgical gloves were worn by the physician before injection. The treatment solution containing $4.75 \mathrm{ml}$ of $0.2 \%$ ropivacaine and $0.25 \mathrm{ml}$ of triamcinolone (a total volume of $5 \mathrm{ml}$ ) was injected for both groups. The needle was advanced when the cartilage was touched slightly under ultrasound imaging. To determine the exact location of the needle, a small volume of the injected solution $(1 \mathrm{ml})$ was initially injected after the initial needle insertion was completed. If the initial $1 \mathrm{ml}$ solution did not enter into the IA space, repositioning of the needle was allowed only once. It was considered as successful when the solution entered into the IA space after repositioning of the needle. Even if the injection was not considered successful, the IA injection proceeded and was completed after repositioning the needle more than once.

All IA injections were performed by one experienced pain physician. We called it a failure of the procedure if the needle was repositioned more than once after the initial placement. All the cases were recorded. The primary outcome was to compare the success rate of two methods of IA injection. The required length of the needle for injection was also measured and compared. Pain intensity was assessed by the NRS in order to evaluate the success of the injection.

The data were expressed as mean \pm SD or numbers (percentages), and statistical analysis was performed using the PASS for the Windows version 14.0.8 (PASS 14 Power Analysis and Sample Size Software (2015). NCSS, LLC. Kaysville, UT) and Stata (Stata/SE 14.2 for Windows, StataCorp LLC, College Station, TX). We used the t-test or chi-square test for comparing the two groups' numer- 

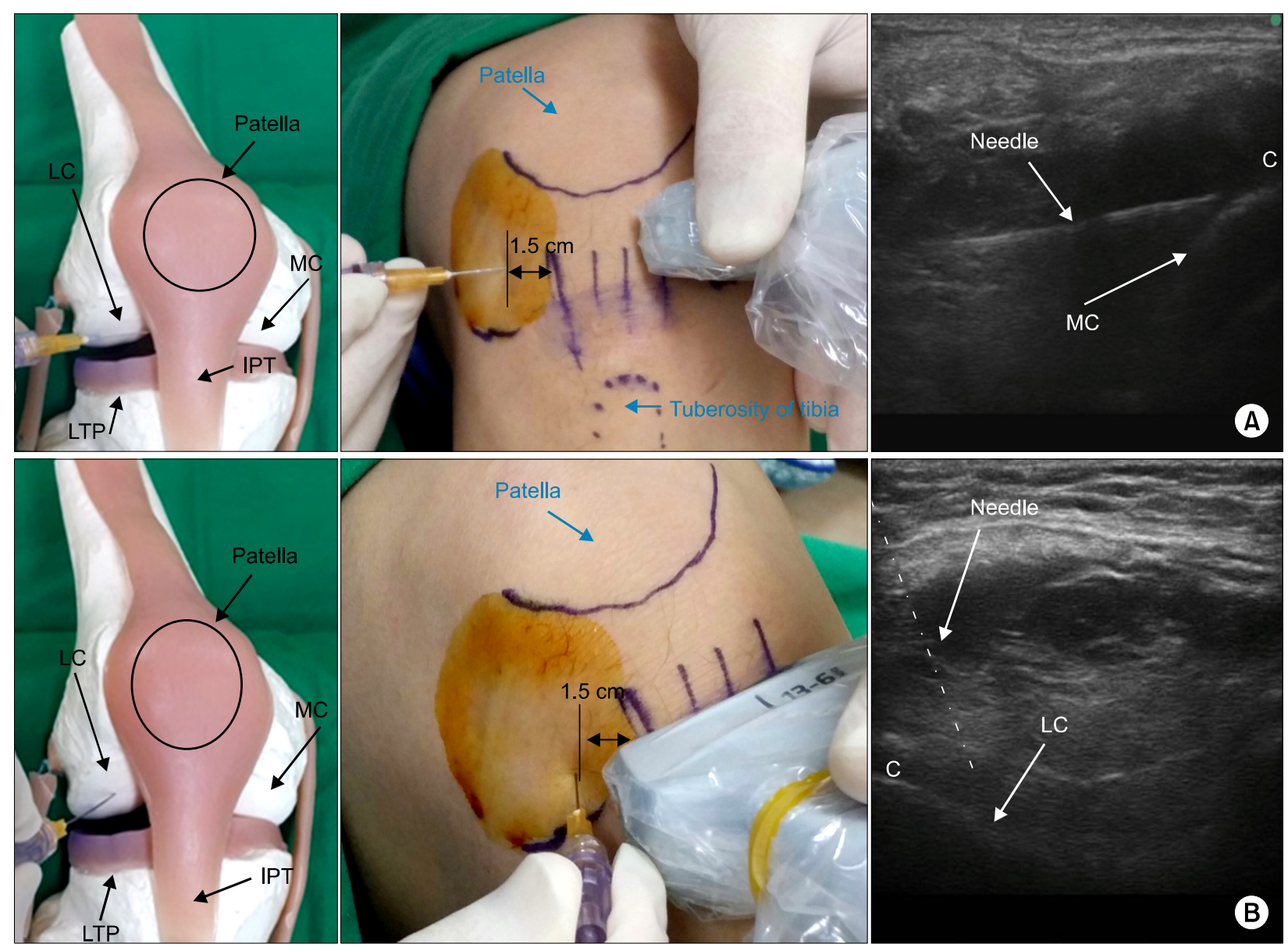

Fig. 2. Photographs and ultrasound image for procedure. The knee was in 90 degrees flexed position. The entry point of the needle is about $1.5 \mathrm{~cm}$ far from the lateral margin of the infrapatellar tendon. The direction of the needle and the target point are shown. (A) Group I: anterolateral approach to the medial condyle. The ultrasound probe was placed over the anteromedial portal so that the ultrasound beam was approximately at the right angles to the needle shaft optimizing visualization of the location of the needle engaged to the medial femoral condyle. (B) Group II: anterolateral approach to the lateral condyle. The ultrasound probe was placed over the central portion of the tibial plateau, and then caudally tilting until a clear lateral condyle image was seen. If the needle was not able be seen, the needle was advanced depending on the movement of tissue planes around the needle. LC: lateral femoral condyle, MC: medial femoral condyle, IPT: infrapatellar tendon, LTP: lateral tibial plateau, C: cartilage.

ical or categorical data, and two-way ANOVA was used for comparing pre- and post-injection pain in each group. $P$ values $<0.05$ were considered statistically significant.

\section{RESULTS}

A total of fifty patients without joint effusion were included in this prospective study. In the study, 96 knees were enrolled. There was no discontinuation of study subjects (Fig. 3). There were no significant differences in the basic characteristics of the study population and knees between the two groups except for the left/right side of the knee (Table 1). The left/right side of the knees were the only statistical difference between the two groups $(P=0.042)$. However, the analysis showed that the left/right knees factor did not affect the success rate.

There was no significant difference in the success rate between the two groups (Table 2). After the needle insertion was initially completed, a true IA injection was only observed in 33\% (16/49) in group I, and 38\% (18/47) in group II (Table 2). However, even if the remaining cases were considered failure, IA injection was successfully per- 


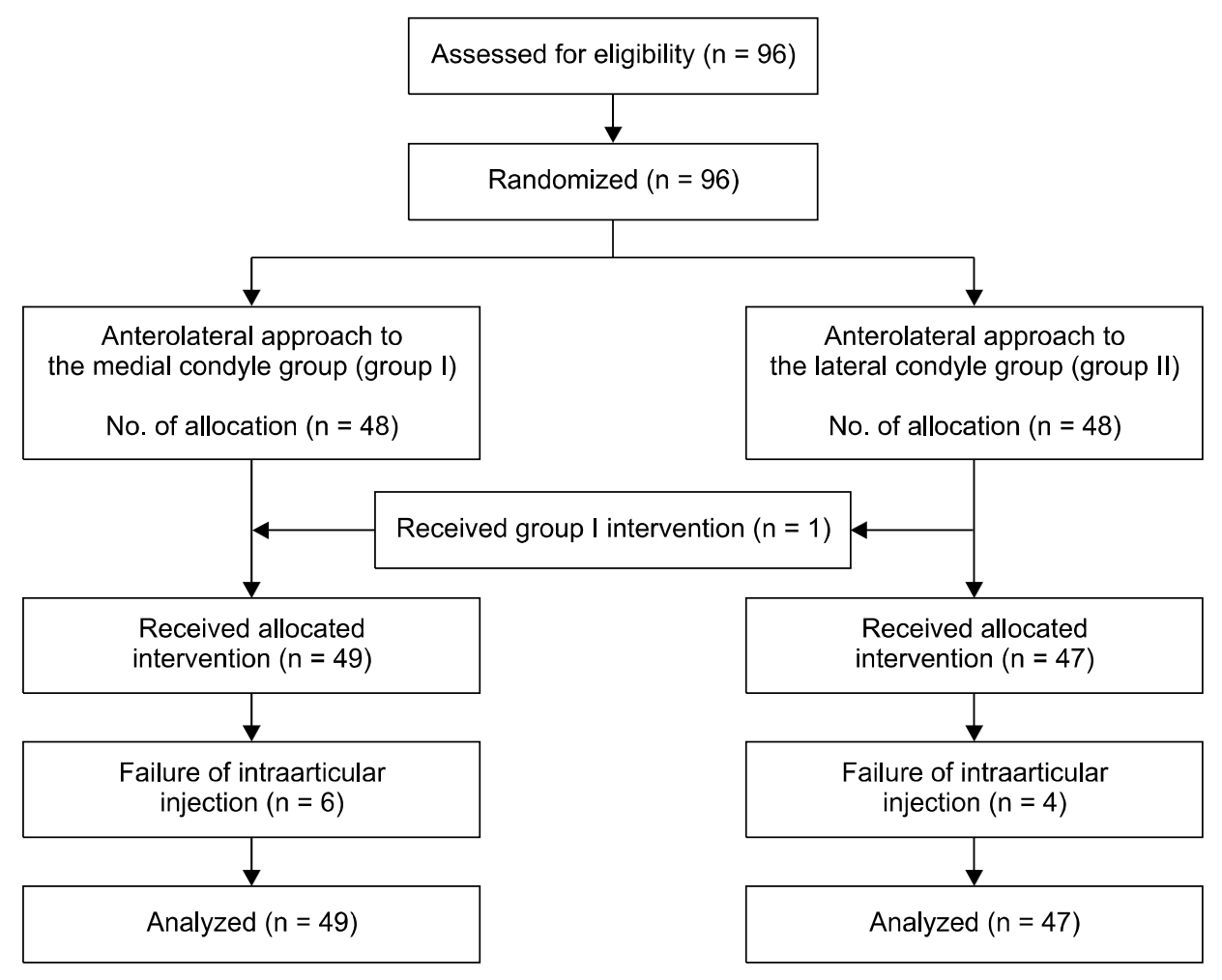

Fig. 3. Flow chart showing the distribution of study patients from initial contact to completion of the study. One case received the injection by group I method due to an error in group II.

Table 1. Basic Characteristic of Study Population and Knee

\begin{tabular}{|c|c|c|c|}
\hline & Group I $(n=49)$ & Group $\|(n=47)$ & $P$ value* \\
\hline Age (years) & $63.2 \pm 18.4$ & $65.7 \pm 17.8$ & 0.508 \\
\hline \multicolumn{4}{|l|}{ Sex } \\
\hline Female & $32(65.3)$ & $30(63.8)$ & 0.880 \\
\hline Male & $17(34.7)$ & $17(36.2)$ & \\
\hline Height $(\mathrm{cm})$ & $160.9 \pm 7.8$ & $159.6 \pm 8.4$ & 0.417 \\
\hline Weight (kg) & $60.6 \pm 10.5$ & $58.6 \pm 10.0$ & 0.346 \\
\hline BMI $\left(\mathrm{kg} / \mathrm{m}^{2}\right)$ & $23.3 \pm 3.5$ & $22.9 \pm 2.9$ & 0.529 \\
\hline \multicolumn{4}{|l|}{ Left/Right } \\
\hline Left & $32(65.3)$ & $21(44.7)$ & 0.042 \\
\hline Right & $17(34.7)$ & $26(55.3)$ & \\
\hline Knee Circumference $(\mathrm{cm})$ & $34.8 \pm 3.1$ & $34.2 \pm 3.1$ & 0.363 \\
\hline
\end{tabular}

Data were presented as mean \pm standard deviation or number (percentage). *Analyzed by t-test or chi-square test.

formed by repositioning the needle more than once. There were significant differences in the measured needle depth between the two groups (Table 2).

The needle depth was $5.0 \pm 0.8$ (range: 3.0 to 6.1 $\mathrm{cm}$ ) in group I, and $3.0 \pm 0.8$ (range: 1.5 to $5 \mathrm{~cm}$ ) in group II $(P<0.001)$. The needle depth of group I was statistically greater than group II $(P<0.001)$. In group I, 21 patients required needles longer than $5.1 \mathrm{~cm}$. There were significant differences between the pre- and post-injection pain in the group (Table 2).

The percentage of reduction from the baseline pain was $64.3 \pm 19.2 \%$ in group I and $64.7 \pm 19.6 \%$ in group II. Bleeding was not observed in either group.

\section{DISCUSSION}

This study showed that there was no difference between the two injection methods when comparing the success 
Table 2. Outcomes of the Intraarticular Injection of the Knee

\begin{tabular}{lccc}
\hline & Group I $(n=49)$ & Group II $(n=47)$ & $P$ value \\
\hline Success rates & $87.8 \%(43 / 49)$ & $91.5 \%(43 / 47)$ & 0.549 \\
Success & \multicolumn{3}{c}{} \\
After initial needling & 16 & 18 \\
After first reposition & 27 & 25 \\
After secondary reposition & 5 & 4 & \\
After third reposition & 1 & 0 & $<0.001$ \\
Needle Depth (cm) & $5.0 \pm 0.8$ & $3.0 \pm 0.8^{*}$ & 0.621 \\
Pre-injection Pain (NRS) & $6.3 \pm 1.6$ & $6.5 \pm 1.6$ & 0.798 \\
Post-injection pain (NRS) & $2.3 \pm 1.4^{\dagger}$ & $2.4 \pm 1.5^{\dagger}$ & 0.925 \\
Percentage of pain reduction from the baseline (\%) & $64.3 \pm 19.2$ & $64.7 \pm 19.6$ & 0.777 \\
Needle Introduction Pain (NRS) & $1.6 \pm 0.7$ & $1.5 \pm 0.7$ & \\
\hline
\end{tabular}

Data were presented as mean \pm standard deviation or number (percentage). Analyzed by t-test or chi-square test. *There was significant difference between both groups. ${ }^{\dagger}$ There was significant difference between pre- and post-injection pain $(P<0.0001)$. After secondary reposition, it is regarded as a failure.

rates of IA injection using different ultrasound-guided anterolateral approaches, and the proposed method was as effective as the other.

The IA injection of the knee using an anterolateral approach requires confirmation of the IA injection more than when using other approaches. Ultrasound can be a good tool for this confirmation. The needle target for the anatomy-guided anterolateral approaches is generally the intercondylar notch, which presents a narrow anatomic target that makes the injection difficult, resulting in only a $71 \%$ to $75 \%$ accuracy rate [5].

Clinical experience has shown that the Hoffa's fat pad is the most common ectopic injection site for a missed IA injection with the anterolateral approach [9]. A hyaluronic acid injection into the anterior fat pad causes pain in the patient lasting for several days. It is necessary to increase the success rate of IA injection using the anterolateral approaches. Fluoroscopy has also been used to increase the success rate for the IA injections [10]. Although the radiation dose may not be significant after a single radiogram, repeated injections with this procedure should be done carefully because of the hazard of the accumulated radiation. Therefore, a method using ultrasound with the possibility of a simultaneous on-screen monitoring of injections has been attempted in the anterolateral approaches for confirming IA injection [6,7].

In group I, the ultrasound probe was placed on the anteromedial portal area, and it made a clearly visible image of the medial condyle by adjusting the tilt of the probe
$[6,7]$. In group II, the ultrasound probe was placed on the infrapatellar ligament close to the tibial plate, and then caudally tilted until a clear lateral condyle image was seen. Therefore, with the ultrasound-guided injection it is difficult to select a constant puncture point and direction using the anatomy-guided method, because it is necessary to adjust the probe to view the target image, and, at the same time, to insert the needle.

In group I, it was not easy to determine the entry point of the needle after adjusting the probe because the distance between the probe and the entry point of the needle was large. Because of the use of $25 \mathrm{G}$ needles in the study, it was also not easy to correct the direction of the needle as the depth of the needle increased. However, in group II, the distance between the probe and the entry point of the needle was closer, and the depth of penetration was shallower. Thus, it was easier to select the entry point of the needle and to maintain its direction. Although, the needle was not visualized on the ultrasound image due to its steep angle in group II, there was no difficulty in recognizing the position of the needle tip in the image by the movement of tissue planes around it [11]. In group II, IA injections were successful in all cases, including those considered as failures.

In group I, needle lengths ranging from $3.0 \mathrm{~cm}$ to 6.1 $\mathrm{cm}(5.0 \pm 0.8)$ were required to reach the IA space. In group II, needle lengths ranging from $1.5 \mathrm{~cm}$ to $5.0 \mathrm{~cm}$ (3.0 \pm 0.8) were required. Chavez-Chiang et al. [6] have suggested that the length of the needle used for IA in- 
jection may influence IA accuracy. It is important to choose a needle of sufficient length to penetrate the IA space. If the selected needle is too short, patients may be dissatisfied because they have to be injected again. Among the $25 \mathrm{G}$ needles, a needle with a short length is easier to handle in a clinical setting. There was no difference in the knee circumference between the groups, although this may affect the length of the needle.

In our study, there were no significant differences in pain reduction and needle introduction pain between the groups. In both groups, the significant pain reduction was seen before and after the IA injection, which may indicate a successful injection. Clinical experience has shown that IA injection of the knee joint is more painful when the injected solution enters Hoffa's fat pad. The test volume used in the IA injections was $1 \mathrm{~mL}$ or less.

There are some limitations to this study. First, the ultrasound-guided method of group I, used as a control, is not a standard method. This method was used as a control method because the IA injections can be visualized. However, the image of the injections spreading only within the joint capsule is reliable enough to confirm a successful IA injection. Second, the needle may not be visualized on the ultrasound in group II because the needle was placed in a location angled steeply to the beam. As demonstrated in group I, a $25 \mathrm{G}$ needle is large enough to the visualized on the ultrasound. If the needle is steeply angled to the beam, the needle movement can be recognized through a review of tissue deformation, where the needle tip position is revealed by the movement of tissue planes around it [11]. The IA injection was not difficult in group I.

In conclusion, the anterolateral approach to the lateral femoral condyle, using the ultrasound, is an alternative method to the approach targeting the medial femoral condyle, using a shorter needle when performing the injection with in-plane technique. Selecting the entry point of the needle and maintaining its direction would be easier when using the anterolateral approach to the lateral femoral condyle.

\section{REFERENCES}

1. Jackson DW, Simon TM, Aberman HM. Symptomatic articular cartilage degeneration: the impact in the new millennium. Clin Orthop Relat Res 2001; (391 Suppl): S14-25.

2. Balazs EA, Denlinger JL. Viscosupplementation: a new concept in the treatment of osteoarthritis. J Rheumatol Suppl 1993; 39: 3-9

3. Leopold SS, Redd BB, Warme WJ, Wehrle PA, Pettis PD, Shott S. Corticosteroid compared with hyaluronic acid injections for the treatment of osteoarthritis of the knee. A prospective, randomized trial. J Bone Joint Surg Am 2003; 85-A: 1197-203.

4. Schumacher HR, Chen LX. Injectable corticosteroids in treatment of arthritis of the knee. Am J Med 2005; 118: 1208-14.

5. Jackson DW, Evans NA, Thomas BM. Accuracy of needle placement into the intra-articular space of the knee. J Bone Joint Surg Am 2002; 84-A: 1522-7.

6. Chavez-Chiang CE, Sibbitt WL Jr, Band PA, Chavez-Chiang NR, DeLea SL, Bankhurst AD. The highly accurate anteriolateral portal for injecting the knee. Sports Med Arthrosc Rehabil Ther Technol 2011; 3: 6.

7. Sibbitt WL Jr, Peisajovich A, Michael AA, Park KS, Sibbitt RR, Band PA, et al. Does sonographic needle guidance affect the clinical outcome of intraarticular injections? J Rheumatol 2009; 36: 1892-902.

8. Qvistgaard E, Kristoffersen H, Terslev L, DanneskioldSamsøe B, Torp-Pedersen S, Bliddal H. Guidance by ultrasound of intra-articular injections in the knee and hip joints. Osteoarthritis Cartilage 2001; 9: 512-7.

9. Esenyel C, Demirhan M, Esenyel M, Sonmez M, Kahraman S, Senel B, et al. Comparison of four different intra- articular injection sites in the knee: a cadaver study. Knee Surg Sports Traumatol Arthrosc 2007; 15: 573-7.

10. Cunnington J, Marshall N, Hide G, Bracewell C, Isaacs J, Platt $P$, et al. A randomized, double-blind, controlled study of ultrasound-guided corticosteroid injection into the joint of patients with inflammatory arthritis. Arthritis Rheum 2010; 62: 1862-9.

11. Chapman GA, Johnson D, Bodenham AR. Visualisation of needle position using ultrasonography. Anaesthesia 2006; 61: $148-58$. 